\title{
BMBF-Klausurwoche (ELSA): Würde und Autonomie als Leitprinzipien in Theorie und Praxis der humanen und außerhumanen Lebenswissenschaften. Ethische, rechtliche und theologische Dimensionen
}

\begin{abstract}
Auf Einladung des moraltheologischen Seminars der Katholisch-Theologischen Fakultät an der Universität Bonn kamen vom 21.-26.2.2011 zwölf Nachwuchswissenschaftler aus den Bereichen Recht, Philosophie, Theologie und Medizin zusammen, um - unterstützt von weiteren zwölf „Experten“ - unter der Leitung von Frau Dr. Heike Baranzke die für aktuelle Problemlagen zentralen Leitbegriffe von „Würde“ und „Autonomie“ in ihren vielfältigen kontextspezifischen Bedeutungsgehalten näher aufzuklären. Diese vom Bundesforschungsministerium geförderte „Klausurwoche“ zeichnete sich durch ein Höchstmaß an Perspektivenvielfalt und Interdisziplinarität insbesondere auch in den vertiefenden Diskussionen aus und kann als vorbildlich für das Gelingen eines interdisziplinären Dialoges gelten. Die Beiträge werden der interessierten Fachöffentlichkeit demnächst in einem von Frau Dr. Baranzke und Prof. Dr. Duttge herausgegebenen Band (,Würde und Autonomie als Leitprinzipien der Bioethik“, Verlag Königshausen \& Neumann, Würzburg) zugänglich gemacht.

Es ist hier nicht der Ort, um die unzähligen Facetten der weitgespannten Thematik auch nur ansatzweise wiedergeben zu können. In insgesamt vier Tagesschwerpunkten wurden sowohl begründungstheoretische Grundsatzfragen aus dem Blickwinkel von Moralphilosophie (Prof. Dr. Birnbacher; Frau Dr. Baranzke), Theologie (Prof. Dr. Wolbert) und Verfassungsrecht (Prof. Dr. Spranger) als auch bedeutsame Anwendungsfelder eingehend behandelt, ehe am Abschlusstag die interkulturelle Anschlussfähigkeit der benannten Leitbegriffe insbesondere im Hinblick auf islamisch geprägte Gesellschaften im Blickpunkt stand (PD Dr. Pink; Prof. Dr. Yildiz zur türkischen Verfassung; Dipl.Jurist Wolf zur UN-Behindertenrechtskonvention). Die methodenkritische Einsicht, sich besser von vermeintlich gesicherten Vorverständnissen reflektierend zu distanzieren (Frau Dr. Dietrich), führte auf den drei mit Bedacht ausgewählten Anwendungsfeldern zu teilweise überraschend neuen Betrachtungsweisen und Erkenntnissen jenseits des hier jeweils begegnenden Diskussionsstandes: So wirft im Kontext des Umgangs mit Patienten am Lebensende die
\end{abstract}

Dr. iur. Markus Thier,

Universität Zürich, Schweiz hohe Relevanz des „Lebensqualitäts“-Paradigmas in der Palliativmedizin (Prof. Voltz) und die hierzu mitunter praktizierte (wenngleich durchaus kritisch zu betrachtende) sog. ,terminale Sedierung“ (Frau M. A. Bozarro) in Zusammenschau mit der Notwendigkeit lebensadäquater „Altersbilder“ (M. A. Schweda) und der „Kommerzialisierungs“Debatte des Transplantationsrechts (Dr. Thier) die Frage auf, ob es nicht jenseits der vorherrschenden ,,autonomiezentrierten" Sichtweise letztlich doch um die Gewährleistung eines „guten Lebens“ (oder „guten Sterbens“, dazu Prof. Dr. Duttge und Frau M.A. Klitzke unter Bezugnahme des „Würde“-Verständnisses bei Robert Spaemann) geht. Im Bereich der neurowissenschaftlichen Herausforderungen wird diese reduktionistische Perspektivenverengung auf die „Autonomie“(-befähigung) des Einzelnen besonders sichtbar, was die Teilnehmer nicht nur theoretisch-konzeptionell (Frau PD Dr. Rehbock; Frau Dr. Dr. Friedrich; Frau M.A. Bittner) und anhand von Fallbeispielen (Frau Dr. Krug zur „Tiefen Hirnstimulation“) kontrovers diskutierten, sondern auch durch Einblicke in den aktuellen Stand der „Neuro-Therapien“ vor Ort im Rahmen einer Führung durch das Institut für Rekonstruktive Neurobiologie (Life \& Brain Center, Bonn) unter der Leitung von Dr. Christian Hoppe (der zugleich fesselnd über die Möglichkeit eines ,aufgeklärten Glaubens in Zeiten der modernen Hirnforschung "vortrug) erfahren durften. Spätestens im Übergang zu Verwendungsweisen des „Würde“-Begriffs im außerhumanen Bereich (z.B. „Würde der Kreatur") wird deutlich, dass seine vielfältigen Bedeutungsdimensionen und deren Korrelationen noch längst nicht hinreichend ausgelotet sind (Prof. Kunzmann; Frau Dr. Odparlik; Frau M.A. Pinsdorf; Frau Dr. Aurenque zur synthetischen Biologie; Dr. Gruber mit dem Gedankenexperiment einer „Phylogenese der Würde").

Die Inkommensurabilität der „Würde“, wie sie abschlieBend am Beispiel des Krankheitsbildes "Demenz" in einer das Publikum ergreifenden Lesung durch Tilman Jens nochmals praktische Anschaulichkeit erlangte, wird erst bei differenzierter Analyse im interdisziplinären Zusammenwirken greifbar. Es stünde dem (Verfassungs- und Medizin-)Recht gut zu Gesicht, diese interdisziplinären Bemühungen und Debatten aufmerksam zu verfolgen und deren Erkenntnisse bei eigenen Explikationen zu Art. 1 GG zu berücksichtigen. 